\title{
On products of eigenforms
}

\author{
by \\ Eknath Ghate (Mumbai)
}

1. Introduction. In [5] we observed that, apart from sixteen $\left({ }^{1}\right)$ exceptions, the product of two level one eigenforms is not an eigenform. This observation was also made independently by W. Duke [4]. The purpose of this note is to investigate whether the product of eigenforms can be an eigenform when the level is square-free.

Let $M_{k}\left(\Gamma_{1}(N)\right)$ denote the space of modular forms of weight $k \geq 1$ and level $N \geq 1$. Let $S_{k}\left(\Gamma_{1}(N)\right)$ denote the subspace of cusp forms, and let $\mathcal{E}_{k}\left(\Gamma_{1}(N)\right)$ denote the subspace of those modular forms that are orthogonal to $S_{k}\left(\Gamma_{1}(N)\right)$ with respect to the Petersson inner product. Thus we have

$$
M_{k}\left(\Gamma_{1}(N)\right)=S_{k}\left(\Gamma_{1}(N)\right) \oplus \mathcal{E}_{k}\left(\Gamma_{1}(N)\right) .
$$

These spaces may be decomposed according to nebentypus:

$$
\begin{aligned}
& M_{k}\left(\Gamma_{1}(N)\right)=\bigoplus_{\chi} M_{k}(N, \chi), \\
& S_{k}\left(\Gamma_{1}(N)\right)=\bigoplus_{\chi} S_{k}(N, \chi), \\
& \mathcal{E}_{k}\left(\Gamma_{1}(N)\right)=\bigoplus_{\chi} \mathcal{E}_{k}(N, \chi),
\end{aligned}
$$

as $\chi$ varies through all Dirichlet characters mod $N$.

Each of the spaces in (1) has an explicit basis consisting of modular forms that are eigenvectors of all the Hecke operators $T_{n}$ with $n$ coprime to $N$.

For $S_{k}(N, \chi)$ this basis is constructed from newforms of lower level in the usual manner. Let $M$ be a positive integer divisible by the conductor $c(\chi)$ of $\chi$. We say that $f \in S_{k}(M, \chi)$ is primitive if it is a newform, it is an eigenvector of all the Hecke operators of level $M$, and it is normalized so

2000 Mathematics Subject Classification: 11F11, 11F67.

$\left({ }^{1}\right)$ In [5] the exceptional cases were miscounted as fifteen; the relation $E_{14}=E_{4} E_{10}$ should have been included in equation (1) of [5]. 
that the $q$-term in its $q$-expansion is 1 . Then the set of cusp forms

$$
\bigcup_{M} \bigcup_{Q}\left\{f(Q z) \mid f \text { is a primitive element of } S_{k}(M, \chi)\right\},
$$

where $M$ varies through all positive integers satisfying $M \mid N$ and $c(\chi) \mid M$, and $Q$ varies through all positive integers dividing $N / M$, forms a basis of $S_{k}(N, \chi)$ consisting of common eigenforms of all the Hecke operators $T_{n}$ with $n$ coprime to $N$.

A similar explicit basis consisting of modular forms that are eigenvectors of all the Hecke operators $T_{n}$ for $n$ coprime to $N$ exists for $\mathcal{E}_{k}(N, \chi)$ as well. We refer the reader to Theorem 2 in the text for its description.

Let $\mathcal{B}$ denote the explicit basis of $M_{k}\left(\Gamma_{1}(N)\right)$ obtained as above. We shall say that an element of $M_{k}\left(\Gamma_{1}(N)\right)$ is an almost everywhere eigenform (or a.e. eigenform for short) if, up to a scalar multiple, it is an element of $\mathcal{B}$.

The purpose of this note is to shed some light on the following question which was asked by D. Prasad:

Question 1. Let $k>l \geq 1$ and $N \geq 1$ be integers. Say $g \in M_{l}\left(\Gamma_{1}(N)\right)$ and $h \in M_{k-l}\left(\Gamma_{1}(N)\right)$ are a.e. eigenforms. When is $g h \in M_{k}\left(\Gamma_{1}(N)\right)$ an a.e. eigenform?

There are three cases to consider:
A. $g \in S_{l}\left(\Gamma_{1}(N)\right)$ and $h \in S_{k-l}\left(\Gamma_{1}(N)\right)$,
B. $g \in S_{l}\left(\Gamma_{1}(N)\right)$ and $h \in \mathcal{E}_{k-l}\left(\Gamma_{1}(N)\right)$,
C. $g \in \mathcal{E}_{l}\left(\Gamma_{1}(N)\right)$ and $h \in \mathcal{E}_{k-l}\left(\Gamma_{1}(N)\right)$.

Since the property of being an a.e. eigenform imposes stringent conditions on the $q$-expansion of a modular form and since the convolution product of $q$-expansions is unlikely to preserve these conditions one expects that the answer to Question 1 is "rarely". This is corroborated by the following theorem, which may be considered a summary of the results proved in the main body of the paper (see Proposition 2 and Theorems 3 and 4).

Theorem 1. Say $N$ is square-free. Let $g$ and $h$ be a.e. eigenforms of weight $l \geq 3$ and $k-l \geq 3$, and nebentypus $\psi$ and $\chi$ respectively. Then:

1. In Case $\mathrm{A}, \mathrm{gh}$ is never an a.e. eigenform.

2. In Case $\mathrm{B}$, assume that $g$ is a newform and $\operatorname{dim} S_{k}^{\text {new }}(N, \psi \chi) \geq 2$. Then $g h$ is not an a.e. eigenform.

3. In Case $\mathrm{C}$, assume that $l \neq k / 2$ and that $\psi$ and $\chi$ are primitive characters. Assume that

$$
\operatorname{dim} S_{k}^{\text {new }}(N, \psi \chi) \geq \begin{cases}1 & \text { when } g h \in \mathcal{E}_{k}(N, \psi \chi), \\ 2 & \text { when } g h \in S_{k}(N, \psi \chi) .\end{cases}
$$

Then $g h$ is not an a.e. eigenform. 
The proof of Theorem 1 uses the Rankin-Selberg method and the fact that when $N$ is square-free the Atkin-Lehner involutions act transitively on the set of Eisenstein series.

We now make some comments on the hypotheses of Theorem 1.

The restriction that the weights of $g$ and $h$ be larger than 3 arises because of technical reasons (Eisenstein series of weight 1 and 2 behave slightly differently than Eisenstein series of weight larger than 3) as well as conceptual ones (the Rankin-Selberg $L$-function may vanish at the point of interest when the weight is 1 or 2 ).

In Case B the theorem is false if $g$ is not a newform. For example if $E_{4}$ is the unique normalized Eisenstein series of level one, $\Delta_{12}$ is the Ramanujan Delta function and $\Delta_{16}$ is the unique cusp form of level one and weight 16 , then the identity

$$
\Delta_{12}(z) \cdot E_{4}(z)=\Delta_{16}(z)
$$

of eigenforms will propagate to $S_{16}\left(\Gamma_{0}(N)\right)$ for arbitrary $N$ by simply substituting $N z$ for $z$. Thus it makes sense to require $g$ to be a newform.

In Case $\mathrm{C}$ we remind the reader that even though $g$ and $h$ are Eisenstein series it is possible that $g h$ is a cusp form. For instance if

$$
g=f_{3}\left(z, \chi_{-3}, 1\right) \in \mathcal{E}_{3}\left(3, \chi_{-3}\right) \quad \text { and } \quad h=f_{5}\left(z, 1, \chi_{-3}\right) \in \mathcal{E}_{5}\left(3, \chi_{-3}\right)
$$

(here $\chi_{-3}$ is the quadratic character of conductor 3 ; see Theorem 2 for the other notation) then $g$ vanishes exactly at the cusp $\infty$ whereas $h$ vanishes exactly at the cusp 0 . Since there are only two cusps for $\Gamma_{1}(3)$ we see that $g h \in S_{8}\left(\Gamma_{0}(3)\right)$ must be a cusp form. (It is in fact an eigenform since $S_{8}\left(\Gamma_{0}(3)\right)$ has dimension 1.)

In Case $\mathrm{C}$ the assumption that $\psi$ and $\chi$ are primitive is made, as in Case B, to exclude from consideration identities in level $N$ which have propagated from smaller levels (example: if $E_{k}$ is the unique normalized Eisenstein series of level one and weight $k$ then $E_{4}(N z) E_{6}(N z)=E_{10}(N z)$ in $\mathcal{E}_{10}\left(\Gamma_{0}(N)\right)$ for all $\left.N\right)$.

The assumption that $l \neq k / 2$ in Case $\mathrm{C}$ is more serious and interesting. It arises since the (twisted) $L$-function of a cusp form of weight $k$ may vanish at the center of the critical strip. In this case the method of proof of this paper fails. However one can work around this in special cases by a direct comparison of Fourier coefficients. When $N=1$ this was done in [5]. When $N$ is arbitrary we only sketch the strategy for doing this in general. As an application of this circle of ideas we prove a result which gives a criterion for the non-vanishing of certain (twisted) standard $L$-functions at the center of the critical strip (see Theorem 5 and its corollaries).

We end this paper with some examples of a.e. eigenforms which can be written as products of a.e. eigenforms of smaller weight. 
2. Rankin-Selberg method. In this section we briefly review the Rankin-Selberg method since it is at the heart of the proof of Theorem 1. A reference is Shimura [9].

Let $N \geq 1$ and $k>l \geq 1$ be integers. Let $\chi$ and $\psi$ be Dirichlet characters $\bmod N$. Say that $f=\sum a_{n} q^{n} \in S_{k}(N, \chi)$ and $g \in M_{l}(N, \psi)$. Let $f_{c}(z)=$ $\overline{f(-\bar{z})}=\sum_{n} \bar{a}_{n} q^{n}$. Then some standard integration shows

$$
I(s, f, g):=\int_{0}^{\infty} \int_{0}^{1} \overline{f_{c}(z)} g(z) y^{s-1} d x d y=(4 \pi)^{-s} \Gamma(s) D(s, f \otimes g),
$$

where

$$
D(s, f \otimes g)=\sum_{n=1}^{\infty} \frac{a_{n} b_{n}}{n^{s}}
$$

is the Rankin product $L$-function of $f$ and $g$. Now let $D=\Gamma_{0}(N) \backslash H$. Then another standard unwinding argument shows that

$$
I(s, f, g)=\iint_{D} \overline{f_{c}(z)} g(z) E_{k-l, \chi \psi}(s+1-k, z) y^{k} \frac{d x d y}{y^{2}},
$$

where

$$
E_{k, \omega}(s, z)=\sum_{\Gamma_{0}(N)_{\infty} \backslash \Gamma_{0}(N)} \frac{\omega(d)}{(c z+d)^{k}|c z+d|^{2 s}}
$$

is the Eisenstein series with parameter $s$. If $f_{1} \in S_{k}\left(\Gamma_{1}(N)\right)$ and $f_{2} \in$ $M_{k}\left(\Gamma_{1}(N)\right)$ let

$$
\left(f_{1}, f_{2}\right)=\iint_{D} \overline{f_{1}(z)} f_{2}(z) y^{k} \frac{d x d y}{y^{2}}
$$

be the usual Petersson inner product of $f_{1}$ and $f_{2}$. Specializing the two expressions above for $I(s, f, g)$ at $s=k-1$ yields the important identity

$$
(4 \pi)^{1-k} \Gamma(k-1) D(k-1, f \otimes g)=\left(f_{c}, g E_{k-l, \chi \psi}\right),
$$

where $E_{k-l, \omega} \in \mathcal{E}_{k-l}(N, \bar{\omega})$ is a modular form (except in the case when both $k-l=2$ and $\omega=1$ ) which is described in more detail in Section 3.

3. Eisenstein series on $\Gamma_{1}(N)$. In this section we recall some facts about Eisenstein series on $\Gamma_{1}(N)$ for an arbitrary integer $N \geq 1$. Further details can be found in Chapters 2, 4, and 7 of Miyake's book [8].

Let $t$ be the number of inequivalent cusps for $\Gamma_{1}(N)$. If $N \geq 3$, then $-1 \notin \Gamma_{1}(N)$, and so we may further classify the cusps as regular or irregular (see page 19 of [8]). Let $u$ denote the number of regular cusps and $v$ the 
number of irregular cusps (for $N \geq 3$ ). We have (cf. [8], Theorem 4.2.9):

$$
t= \begin{cases}1 & \text { if } N=1, \\ 2 & \text { if } N=2, \\ 2 & \text { if } N=3(0 \text { and } \infty \text { are both regular), } \\ 3 & \text { if } N=4(0, \infty \text { are regular, } 1 / 2 \text { is irregular), } \\ \frac{1}{2} \sum_{0<d \mid N} \phi(d) \phi(N / d) & \text { if } N \geq 5 \text { (all cusps are regular). }\end{cases}
$$

The number of cusps is closely connected to the dimension of the space of Eisenstein series. In fact we have (cf. [8], Theorems 2.5.2 and 2.5.3):

$$
\operatorname{dim} \mathcal{E}_{k}\left(\Gamma_{1}(N)\right)= \begin{cases}t & \text { if } k \geq 4 \text { is even, } \\ t-1 & \text { if } k=2, \\ u & \text { if } k \geq 3 \text { is odd and } N \geq 3, \\ u / 2 & \text { if } k=1 \text { and } N \geq 3 \\ 0 & \text { if } k \geq 1 \text { is odd and } N=1,2 .\end{cases}
$$

Note that in the generic case, namely $k \geq 3$ and $N \geq 5$, the dimension is

$$
t=u=\frac{1}{2} \sum_{0<d \mid N} \phi(d) \phi(N / d) .
$$

In fact it is easy to explicitly write down a basis of a.e. eigenforms of $\mathcal{E}_{k}\left(\Gamma_{1}(N)\right.$ ) (see [8], Theorems 4.7.1 and 4.7.2):

THEOREM 2. Let $\chi_{1}$ and $\chi_{2}$ be Dirichlet characters mod $M_{1}$, respectively $M_{2}$, such that $\chi_{1} \chi_{2}(-1)=(-1)^{k}$ with $k \geq 1$. Assume that

- if $k=2$ and $\chi_{1}=\chi_{2}=1$, then $M_{1}=1$ and $M_{2}$ is a prime number,

- otherwise, $\chi_{1}$ and $\chi_{2}$ are primitive characters.

Then there is an element $f=f_{k}\left(z, \chi_{1}, \chi_{2}\right)=\sum_{n=0}^{\infty} a_{n} q^{n} \in \mathcal{E}_{k}(M, \chi)$, with $M=M_{1} M_{2}$ and $\chi=\chi_{1} \chi_{2}$, characterized by the properties

- $L(s, f)=L\left(s, \chi_{1}\right) L\left(s-k+1, \chi_{2}\right)$, and

- the constant term of $f$ is

$$
a_{0}= \begin{cases}0 & \text { if } k \geq 2 \text { and } \chi_{1} \neq 1, \text { or } \\ & \text { if } k=1 \text { and both } \chi_{1} \neq 1 \text { and } \chi_{2} \neq 1 \\ (M-1) / 24 & \text { if } k=2 \text { and } \chi_{1}=1, \chi_{2}=1 \\ -B_{k, \chi} /(2 k) & \text { otherwise. }\end{cases}
$$

The modular form $f_{k}\left(z, \chi_{1}, \chi_{2}\right) \in \mathcal{E}_{k}(M, \chi)$ is an eigenvector for all the Hecke operators of level $M$. Modulo the relation $f_{1}\left(z, \chi_{1}, \chi_{2}\right)=f_{1}\left(z, \chi_{2}, \chi_{1}\right)$ when $k=1$, the set of elements

$$
f_{k}\left(Q z, \chi_{1}, \chi_{2}\right)
$$

with $Q M_{1} M_{2} \mid N$, and $\chi_{1}, \chi_{2}, M_{1}, M_{2}$ satisfying the conditions above, form a basis of $\mathcal{E}_{k}(N, \chi)$ consisting of common eigenforms of all the Hecke operators $T_{n}$ of level $N$, with $n$ coprime to $N$. 
We leave it to the reader to check (cf. pages 179-181 of [8]) that, by an explicit counting argument, one recovers $\left({ }^{2}\right)$ the formula (4).

Not all the Eisenstein series $f_{k}\left(Q z, \chi_{1}, \chi_{2}\right) \in \mathcal{E}_{k}(N, \chi)$ of Theorem 2 appear in the Rankin-Selberg method. In fact those that do are essentially those coming from the cusp at $\infty$. Let us make this more precise. Fix an integer $k \geq 1$ and a Dirichlet character $\omega \bmod N$. Then the Eisenstein series that appear in the Rankin-Selberg method are of the form

$$
E_{k, \omega}(z)=\sum_{\Gamma_{0}(N)_{\infty} \backslash \Gamma_{0}(N)} \frac{\omega(d)}{(c z+d)^{k}}=\frac{1}{2} \sum_{\substack{c \equiv 0(N) \\(c, d)=1}} \frac{\omega(d)}{(c z+d)^{k}} .
$$

Except for the case $k=2$ and $\omega=1, E_{k, \omega}(z)$ is a holomorphic function of $z$, and so defines an element

$$
E_{k, \omega} \in M_{k}(N, \bar{\omega}) .
$$

Actually, when $k=1$ or 2 (and $\omega$ is arbitrary) the series (5) may not converge absolutely. It is defined instead by a well known trick of Hecke: one considers the Eisenstein series in (2),

$$
E_{k, \omega}(s, z)=\sum_{\Gamma_{0}(N)_{\infty} \backslash \Gamma_{0}(N)} \frac{\omega(d)}{(c z+d)^{k}|c z+d|^{2 s}},
$$

shows that it has an analytic continuation to a right half plane containing $s=0$, and then one sets

$$
E_{k, \omega}(z)=E_{k, \omega}(0, z) .
$$

When $k=2$ and $\omega=1$, the specialization $E_{2,1}(z)$ so obtained is nonholomorphic in $z$. However one knows that for each prime $p$,

$$
E_{2}^{(p)}(z)=E_{2,1}(z)-p E_{2,1}(p z)
$$

is a holomorphic function of $z$, and so defines an element of $M_{2}(N, 1)$ for each $p \mid N$. Further details can be found in Chapter 7 of [8].

We now identify the Eisenstein series that appear in the Rankin-Selberg method in terms of the basis of a.e. eigenforms given in Theorem 2:

$\left({ }^{2}\right)$ There are some minor typos in the formula for the dimension of $\mathcal{E}_{k}\left(\Gamma_{1}(N)\right)$ on page 181 of [8]. The second entry should read $k=2$, rather than $k \geq 2$. Moreover the first two entries of the formula do not make sense when $N=4$, and should be replaced by

$$
\operatorname{dim} \mathcal{E}_{k}\left(\Gamma_{1}(4)\right)= \begin{cases}3 & \text { if } k \geq 4 \text { is even } \\ 2 & \text { if } k \geq 3 \text { is odd } \\ 2 & \text { if } k=2\end{cases}
$$

as predicted by the formula (4). 
Lemma 1. 1. Assume that $k \geq 3$. We have

$$
E_{k, \omega}(z)=-\frac{2 k}{B_{k, \bar{\omega}}} \cdot f_{k}\left(Q z, \chi_{0}, \bar{\chi}_{2}\right),
$$

where $\chi_{0}$ is the principal character of level $M_{1}=1, \chi_{2}$ is the primitive character of conductor $M_{2}$ associated to $\omega$, and $Q=N / M_{2}$.

2. When $k=2$ and $\omega=1$, we have

$$
E_{2}^{(p)}(z)=-8 \pi^{2} \cdot f_{2}\left(z, \chi_{0}, 1_{p}\right)
$$

where $1_{p}$ is the trivial character of level $M_{2}=p$.

Proof. Assume momentarily that $\omega$ is primitive of level N. By (7.1.13) and (7.1.30) of [8], we see that

$$
E_{k, \omega}(z)=-\frac{2 k}{B_{k, \omega}} \cdot f_{k}\left(z, \chi_{0}, \bar{\omega}\right)
$$

as desired. The constant of proportionality was computed using Theorem 3.3.4(1) of [8]. More generally, if $\omega$ is associated to the primitive character $\chi_{2}$ of level $M_{2}$, then substituting $c=c^{\prime} Q$ with $Q=N / M_{2}$ in (5) above, we have

$$
E_{k, \omega}(z)=\frac{1}{2} \sum_{\substack{c^{\prime} \equiv 0\left(M_{2}\right) \\\left(c^{\prime}, d\right)=1}} \frac{\chi_{2}(d)}{\left(c^{\prime} Q z+d\right)^{k}}=E_{k, \chi_{2}}(Q z)=-\frac{2 k}{B_{k, \bar{\chi}_{2}}} \cdot f_{k}\left(Q z, \chi_{0}, \bar{\chi}_{2}\right)
$$

since $(Q, d) \neq 1 \Rightarrow(N, d) \neq 1 \Rightarrow \omega(d)=0$. This proves the first statement. Actually the proof given here should work for all $k \geq 1$ except the case when $k=2$ and $\omega=1$ but we do not pursue this point here.

For the second statement we simply refer the reader to Lemma 7.2.19 of $[8]$.

Let us close this section with the following corollary to Theorem 2:

Corollary 1. The tuples $(k, N, \chi)$ for which $\operatorname{dim} \mathcal{E}_{k}(N, \chi)=1$, along with an explicit generator, are as follows (here $p$ denotes a prime):

Table 1. Tuples $(k, N, \chi)$ with $\operatorname{dim} \mathcal{E}_{k}(N, \chi)=1$

\begin{tabular}{|c|c|c|c|}
\hline Weight & Level & Nebentypus & Generator \\
\hline$k=1$ & $N=p^{m}, N \neq 2$ & $\chi$ is primitive, odd & $E_{1, \chi}$ \\
$k=2$ & $N=p$ & $\chi=1$ & $E_{2}^{(p)}$ \\
$k \geq 4$ is even & $N=1$ & $\chi=1$ & $E_{k, \chi_{0}}$ \\
\hline
\end{tabular}

4. Atkin-Lehner involutions. Let $N$ be an arbitrary integer, and let $Q \mid N$ be such that

$$
(Q, N / Q)=1
$$


Then there is an Atkin-Lehner involution $w_{Q}$ defined by

$$
w_{Q}=\left(\begin{array}{cc}
Q x & y \\
N v & Q w
\end{array}\right)
$$

where $x, y, v, w \in \mathbb{Z}$ satisfy $x \equiv 1 \bmod N / Q, y \equiv 1 \bmod Q$ and $\operatorname{det}\left(w_{Q}\right)=Q$. $w_{Q}$ acts on the space $M_{k}(N, \chi)$ in the usual way: if $f \in M_{k}(N, \chi)$ then

$$
\left.f\right|_{w_{Q}}(z)=\operatorname{det}\left(w_{Q}\right)^{k / 2} j\left(w_{Q}, z\right)^{-k} f\left(w_{Q} z\right) .
$$

Decompose $\chi=\chi_{Q} \cdot \chi_{N / Q}$ into its $Q$ and $N / Q$ parts. It is well known (see Proposition 1.1 of [1]) that the Atkin-Lehner involution $w_{Q}$ maps

$$
w_{Q}: M_{k}\left(N, \chi_{Q} \cdot \chi_{N / Q}\right) \rightarrow M_{k}\left(N, \bar{\chi}_{Q} \cdot \chi_{N / Q}\right)
$$

and that it takes cusp forms to cusp forms. Moreover, since $w_{Q}$ commutes, up to a constant, with $T_{n}$ for all $n$ with $(n, N)=1$, it takes a.e. eigenforms to a.e. eigenforms (see Proposition 1.2 of [1]). In fact $w_{Q}$ takes primitive cusp forms (of level $N$ ) to primitive cusp forms (up to multiplication by a constant). On the other hand the following proposition describes how the a.e. eigenforms of Theorem 1 are permuted under the $w_{Q}$.

Proposition 1. Let $k \geq 3$. Let $f_{k}\left(z, \chi_{1}, \chi_{2}\right) \in \mathcal{E}_{k}(N, \chi)$ be an a.e. eigenform as in Theorem 2. Let $w_{Q}$ be the Atkin-Lehner involution defined in (7). Write $Q_{1}=\left(Q, M_{1}\right)$ and $Q_{2}=\left(Q, M_{2}\right)$ and decompose

$$
\chi_{1}=\chi_{Q_{1}} \chi_{M_{1} / Q_{1}} \quad \text { and } \quad \chi_{2}=\chi_{Q_{2}} \chi_{M_{2} / Q_{2}}
$$

into their $Q$ and prime-to- $Q$ parts. Set

$$
\alpha=\frac{\chi_{Q_{2}}\left(-M_{2} / Q_{2}\right) \chi_{M_{2} / Q_{2}}\left(Q_{2}\right)}{\chi_{Q_{1}}\left(M_{2} / Q_{2}\right) \chi_{M_{1} / Q_{1}}\left(Q_{2}\right)} .
$$

Then

$$
\left.f_{k}\left(z, \chi_{1}, \chi_{2}\right)\right|_{w_{Q}}=Q^{k / 2} Q_{2}^{-k} \cdot \alpha \cdot f_{k}\left(\frac{Q z}{Q_{1} Q_{2}}, \bar{\chi}_{Q_{2}} \chi_{M_{1} / Q_{1}}, \bar{\chi}_{Q_{1}} \chi_{M_{2} / Q_{2}}\right) .
$$

Proof. For the primitive characters $\chi_{1}$ and $\chi_{2}$ as above consider the Eisenstein series

$$
E_{k}\left(z, \chi_{1}, \chi_{2}\right)=\sum_{c, d} \frac{\chi_{1}(c) \chi_{2}(c)}{(c z+d)^{k}},
$$

where the sum is over all non-zero integers $c$ and $d$. By (7.1.13) of [8] we have

$$
f_{k}\left(z, \chi_{1}, \chi_{2}\right)=(\text { const }) \cdot E_{k}\left(M_{2} z, \chi_{1}, \bar{\chi}_{2}\right)
$$

We compute 


$$
\begin{aligned}
\left.f_{k}\left(z, \chi_{1}, \bar{\chi}_{2}\right)\right|_{w_{Q}} \\
=Q^{k / 2} j\left(w_{Q}, z\right)^{-k} f\left(w_{Q} z, \chi_{1}, \chi_{2}\right) \\
=Q^{k / 2}(\text { const }) \cdot(N v z+Q w)^{-k} E_{k}\left(M_{2} w_{Q} z, \chi_{1}, \chi_{2}\right) \\
=Q^{k / 2}(\text { const }) \cdot(N v z+Q w)^{-k} \sum_{c, d} \frac{\chi_{1}(c) \chi_{2}(d)}{\left(c M_{2} \frac{Q x z+y}{N v z+Q w}+d\right)^{k}} \\
=Q^{k / 2}(\text { const }) \cdot \sum_{c, d} \frac{\chi_{1}(c) \chi_{2}(d)}{Q_{2}^{k}\left(\left(c x Q_{2}+d \frac{N Q_{2}}{M_{2} Q} v\right)\left(\frac{M_{2} Q z}{Q_{2}^{2}}\right)+\left(c \frac{M_{2}}{Q_{2}} y+d \frac{Q}{Q_{2}} w\right)\right)^{k}} .
\end{aligned}
$$

Make the change of variables

$$
\left(\begin{array}{l}
c^{\prime} \\
d^{\prime}
\end{array}\right)=\left(\begin{array}{cc}
Q_{2} x & \frac{N Q_{2}}{M_{2} Q} v \\
\frac{M_{2}}{Q_{2}} y & \frac{Q}{Q_{2}} w
\end{array}\right)\left(\begin{array}{l}
c \\
d
\end{array}\right) .
$$

Then the change of variables matrix has determinant $Q x w-\frac{N}{Q} v y=1$. Moreover $c^{\prime} \equiv c Q_{2} \bmod M_{1} / Q_{1}$ since $M_{1} / Q_{1} \mid\left(N Q_{2}\right) /\left(Q M_{2}\right)$ and $x \equiv 1 \bmod M_{1} / Q_{1}$, $c^{\prime} \equiv-d Q_{2} / M_{2} \bmod Q_{2}$ since $-N v / Q \equiv 1 \bmod Q_{2}$, $d^{\prime} \equiv c M_{2} / Q_{2} \bmod Q_{1}$ since $Q_{1} \mid Q / Q_{2}$ and $y \equiv 1 \bmod Q_{1}$, and $d^{\prime} \equiv d / Q_{2} \bmod M_{2} / Q_{2}$ since $Q w \equiv 1 \bmod M_{2} / Q_{2}$.

The above congruences imply that

$$
\begin{aligned}
\chi_{1}(c) & =\chi_{Q_{1}}(c) \cdot \chi_{M_{1} / Q_{1}}(c) \\
& =\chi_{Q_{1}}\left(d^{\prime}\right) \chi_{Q_{1}}\left(M_{2} / Q_{2}\right)^{-1} \cdot \chi_{M_{1} / Q_{1}}\left(c^{\prime}\right) \chi_{M_{1} / Q_{1}}\left(Q_{2}\right)^{-1}, \\
\chi_{2}(d) & =\chi_{Q_{2}}(d) \cdot \chi_{M_{2} / Q_{2}}(d) \\
& =\chi_{Q_{2}}\left(c^{\prime}\right) \chi_{Q_{2}}\left(-M_{2} / Q_{2}\right) \cdot \chi_{M_{2} / Q_{2}}\left(d^{\prime}\right) \chi_{M_{2} / Q_{2}}\left(Q_{2}\right) .
\end{aligned}
$$

Thus we have

$$
\begin{aligned}
\left.f_{k}\left(z, \chi_{1}, \bar{\chi}_{2}\right)\right|_{w_{Q}} \\
=Q^{k / 2} Q_{2}^{-k} \alpha(\text { const }) \cdot \sum_{c^{\prime}, d^{\prime}} \frac{\chi_{Q_{1}}\left(d^{\prime}\right) \chi_{M_{1} / Q_{1}}\left(c^{\prime}\right) \chi_{Q_{2}}\left(c^{\prime}\right) \chi_{M_{2} / Q_{2}}\left(d^{\prime}\right)}{\left(c^{\prime} \frac{M_{2} Q z}{Q_{2}^{2}}+d^{\prime}\right)^{k}} \\
=Q^{k / 2} Q_{2}^{-k} \alpha(\text { const }) \cdot E_{k}\left(\frac{Q M_{2} z}{Q_{2}^{2}}, \chi_{Q_{2}} \chi_{M_{1} / Q_{1}}, \chi_{Q_{1}} \chi_{M_{2} / Q_{2}}\right) \\
=Q^{k / 2} Q_{2}^{-k} \alpha \cdot f_{k}\left(\frac{Q z}{Q_{1} Q_{2}}, \chi_{Q_{2}} \chi_{M_{1} / Q_{1}}, \overline{\chi_{Q_{1}} \chi_{M_{2} / Q_{2}}}\right),
\end{aligned}
$$

where the last line follows from (8). Replacing $\chi_{2}$ with $\bar{\chi}_{2}$ yields the proposition. 
5. Case A. In this section we observe that when the level is square-free the product of two cuspidal a.e. eigenforms is never an a.e. eigenform. We have:

Proposition 2. Say that $k>l \geq 1$ are integers and that $N \geq 1$ is a square-free integer. Say $g \in S_{l}\left(\Gamma_{1}(N)\right)$ and $h \in S_{k-l}\left(\Gamma_{1}(N)\right)$ are a.e. eigenforms. Then $g h$ is NOT an a.e. eigenform.

Proof. Say that

$$
g h=f
$$

is an a.e. eigenform. Write $f(z)=f_{0}(Q z)$ where $M|N, Q|(N / M)$ and $f_{0} \in S_{k}(M, \chi)$ is a primitive element. Since $N$ is square-free, condition (6) is automatically satisfied for any divisor $Q$ of $N$. Let $w_{Q}$ be the Atkin-Lehner involution defined in (7). We compute

$$
\begin{aligned}
\left.f\right|_{w_{Q}} & =Q^{k / 2}(N v z+Q w)^{-k} f\left(\frac{Q x z+y}{N v z+Q w}\right) \\
& =Q^{-k / 2}(N v z / Q+w)^{-k} f_{0}\left(\frac{Q x z+y}{N v z / Q+w}\right) \\
& =Q^{-k / 2} \chi(w) f_{0}(z),
\end{aligned}
$$

since

$$
\left(\begin{array}{cc}
Q x & y \\
N v / Q & w
\end{array}\right) \in \Gamma_{0}(M)
$$

Thus, applying $w_{Q}$ to (9) we get $\left.\left.g\right|_{w_{Q}} h\right|_{w_{Q}}=f_{0}$ up to a constant. Clearly this is impossible: both $\left.g\right|_{w}$ and $\left.h\right|_{w}$ are cusp forms so that the $q$-expansion of their product begins with $q^{2}$; on the other hand since $f_{0}$ is primitive its $q$-expansion begins with $q$.

6. Case B. Fix integers $k>l \geq 1$ and $N \geq 1$, and $\bmod N$ characters $\psi$ and $\omega$. Suppose that $g \in S_{l}(N, \psi)$ and $h \in \mathcal{E}_{k-l}(N, \chi)$ are a.e. eigenforms. We wish to know whether $g h$ is an a.e. eigenform. We first investigate the case where $h=E_{k-l, \omega}$ is the Eisenstein series in (5).

Proposition 3. Suppose that $l \geq 1$ and $k-l \geq 3$. Say that $g \in S_{l}(N, \psi)$ is an a.e. eigenform and that $h=E_{k-l, \omega} \in \mathcal{E}_{k-l}(N, \bar{\omega})$. If $g$ is a newform and $\operatorname{dim} S_{k}^{\text {new }}(N, \psi \bar{\omega}) \geq 2$, then $g h$ is NOT an a.e. eigenform.

Proof. First note that the map

$$
f(z)=\sum_{n} a_{n} q^{n} \mapsto f_{c}(z)=\overline{f(-\bar{z})}=\sum_{n} \bar{a}_{n} q^{n}
$$

induces a bijection between the primitive forms in $S_{k}(N, \bar{\psi} \omega)$ and those in $S_{k}(N, \psi \bar{\omega})$. Now suppose, towards a contradiction, that $g h$ is an a.e. eigenform. Then, by the hypothesis on the dimension of $S_{k}(N, \psi \bar{\omega})$, we may 
pick a primitive form $f_{c} \in S_{k}(N, \psi \bar{\omega})$ distinct from $g h$. The Rankin-Selberg method (see (3)) yields

$$
\Gamma(k-1)(4 \pi)^{1-k} D(k-1, f \otimes g)=\left(f_{c}, g E_{k-l, \omega}\right)
$$

where $D(s, f \otimes g)$ is the Rankin product $L$-function of $f$ and $g$. The right hand side vanishes, by choice of $f_{c}$. On the other hand the left hand side does not. Indeed, since $f$ and $g$ are primitive, $D(s, f, g)$ has an Euler product decomposition which converges absolutely in the region $\Re(s)>(k+l) / 2$ and $k-1$ is in this region since $k-l \geq 3$.

Theorem 3. Suppose that $l \geq 3$ and $k-l \geq 3$. Let $g \in S_{l}(N, \psi)$ and $h \in \mathcal{E}_{k-l}(N, \chi)$ be a.e. eigenforms. Assume that $N$ is square-free and that $g$ is a newform. If $\operatorname{dim} S_{k}^{\text {new }}(N, \psi \chi) \geq 2$ then $g h$ is NOT an a.e. eigenform.

Proof. In the notation of Theorem 2 we may write $h=f_{k-l}\left(Q z, \chi_{1}, \chi_{2}\right)$, where $Q \mid N / M_{1} M_{2}$. Since $N$ is square-free, condition (6) is automatically satisfied for any divisor of $N$. In particular let $w=w_{N / Q M_{2}}$ be the AtkinLehner involution defined in Section 4. By Proposition 1 and Lemma 1 we have

$$
\left.h\right|_{w}=\left.f_{k}\left(Q z, \chi_{1}, \chi_{2}\right)\right|_{w_{N / Q M_{2}}}=f_{k}\left(\frac{N z}{M_{1} M_{2}}, \chi_{0}, \bar{\chi}_{1} \chi_{2}\right)=E_{k-l, \chi_{1} \bar{\chi}_{2}},
$$

where $\chi_{0}$ is the principal character and all equalities hold up to a constant.

The formula (11) allows us to reduce the proof of the theorem to Proposition 3. Indeed, suppose, towards a contradiction, that $g h$ is an a.e. eigenform. Since the Atkin-Lehner involutions take a.e. eigenforms to a.e. eigenforms, $\left.\left.g\right|_{w} h\right|_{w}$ is an a.e. eigenform. Admittedly, it lies in the twisted space $S_{k}\left(N, \psi_{Q M_{2}} \bar{\psi}_{N / Q M_{2}} \cdot \bar{\chi}_{1} \chi_{2}\right)$, which nonetheless still satisfies

$$
\operatorname{dim} S_{k}^{\text {new }}\left(N, \psi_{Q M_{2}} \bar{\psi}_{N / Q M_{2}} \cdot \bar{\chi}_{1} \chi_{2}\right) \geq 2 .
$$

Applying Proposition 3 (with $g$ replaced by $\left.g\right|_{w}, \psi$ by $\psi_{Q M_{2}} \cdot \bar{\psi}_{N / Q M_{2}}$, and $\omega=\chi_{1} \bar{\chi}_{2}$ ) shows that this is impossible since $\left.g\right|_{w}$ is a newform. This completes the proof of the theorem.

7. Case C. Fix integers $k>l \geq 1$, and $\bmod N$ Dirichlet character $\psi$ and $\omega$. Suppose that $g \in \mathcal{E}_{l}(N, \psi)$ and $h \in \mathcal{E}_{k-l}(N, \chi)$ are a.e. eigenforms. We wish to know whether $g h$ is an a.e. eigenform.

As in Case B, we begin our discussion with the case when $h=E_{k-l, \omega}$ is the Eisenstein series in (5).

Proposition 4. Suppose that $k>l \geq 1$. Let $g=f_{l}\left(z, \psi_{1}, \psi_{2}\right) \in \mathcal{E}_{l}(N, \psi)$ be an a.e. eigenform as in Theorem 2 with $\psi=\psi_{1} \psi_{2}$ primitive. Let $h=$ $E_{k-l, \omega} \in \mathcal{E}_{k-l}(N, \bar{\omega})$ with $\omega \neq 1$ when $k-l=2$. Assume that

$$
\operatorname{dim} S_{k}^{\text {new }}(N, \psi \bar{\omega}) \geq \begin{cases}1 & \text { if } g h \in \mathcal{E}_{k}(N, \psi \bar{\omega}), \\ 2 & \text { if } g h \in S_{k}(N, \psi \bar{\omega}) .\end{cases}
$$


Assume additionally that one of the following conditions hold:

1. $k-l \geq l+1$,

2. $k \geq 3$ and there is a primitive form $f \in S_{k}(N, \bar{\psi} \omega)$ such that $L(k / 2$, $\left.f \otimes \psi_{2}\right) \neq 0$,

3. $k=2$ and there is a primitive form $f \in S_{2}(N, \bar{\psi} \omega)$ such that both $L\left(1, f \otimes \psi_{1}\right) \neq 0$ and $L\left(1, f \otimes \psi_{2}\right) \neq 0$.

Then $g h \in M_{k}(N, \psi \bar{\omega})$ is NOT an a.e. eigenform.

Proof. Suppose, towards a contradiction, that $g h \in M_{k}(N, \psi \bar{\omega})$ is an a.e. eigenform. Then it would have to lie in either $\mathcal{E}_{k}(N, \psi \bar{\omega})$ or in $S_{k}(N, \psi \bar{\omega})$. In either case, the hypothesis (12) on the dimension of $S_{k}^{\text {new }}(N, \psi \bar{\omega})$ allows us to pick a primitive eigenform $f_{c} \in S_{k}(N, \psi \bar{\omega})$ distinct from $g h$, which is the complex conjugate of a primitive eigenform $f \in S_{k}(N, \bar{\psi} \omega)$. As usual (see (3)) we have the identity

$$
\Gamma(k-1)(4 \pi)^{1-k} D(k-1, f, g)=\left(f_{c}, g E_{k-l, \omega}\right) .
$$

Since we have assumed that $\psi$ is primitive, $g$ is a common eigenform of all the Hecke operators. Thus $D(s, f, g)$ has an Euler product expansion which yields

$$
D(k-1, f, g)=\frac{L\left(k-1, f \otimes \psi_{1}\right) L\left(k-l, f \otimes \psi_{2}\right)}{L(k-l, \omega)},
$$

where the equality is up to some bad Euler factors.

For reasons of parity $k-l=1 \Rightarrow \omega \neq 1$. So the denominator of the right hand side of (14) is a non-zero finite number.

An Euler product argument shows that $L(s, f \otimes \theta) \neq 0$ in the region $\Re(s)>(k+1) / 2$ for any mod $N$ Dirichlet character $\theta$. By Proposition 2 of [9] one can actually extend this slightly: one has $L((k+1) / 2, f \otimes \theta) \neq 0$ as well.

Using this we see that if any of the hypothesis 1,2 or 3 of Proposition 4 hold, then the numerator on the right hand side of (14) is non-zero as well, for a suitable choice of $f$. For instance $k-l \geq l+1$ implies that $k-l \geq(k+1) / 2$ and that $k-1 \geq(k+1) / 2$ so that both the $L$-values in the numerator do not vanish. This yields the desired contradiction since the right hand side of (13) vanishes.

Theorem 4. Suppose that $l \geq 3$ and $k-l \geq 3$. Let $g=f_{l}\left(z, \psi_{1}, \psi_{2}\right) \in$ $\mathcal{E}_{l}(N, \psi)$ and $h=f_{k-l}\left(z, \chi_{1}, \chi_{2}\right) \in \mathcal{E}_{k-l}(N, \chi)$ be a.e. eigenforms as in Theorem 2 with $\psi$ and $\chi$ primitive. Assume that $N$ is square-free and that $l \neq k / 2$. If

$$
\operatorname{dim} S_{k}^{\text {new }}(N, \psi \chi) \geq \begin{cases}1 & \text { when } g h \in \mathcal{E}_{k}(N, \psi \chi), \\ 2 & \text { when } g h \in S_{k}(N, \psi \chi),\end{cases}
$$

then $g h$ is NOT an a.e. eigenform. 
Proof. As in the proof of Theorem 3 we may twist $h$ by the Atkin-Lehner involution $w=w_{N / M_{2}}$ (note that now $Q=1$ ) to assume that $h=E_{k-l, \chi_{1} \bar{\chi}_{2}}$. If $k-l \geq l+1$, then since $\left.g\right|_{w}$ would continue to have primitive nebentypus, the theorem would follow from Proposition 4 . If $k-l \nsupseteq l+1$, switch the roles of $g$ and $h$ (note we are assuming that both $\chi$ and $\psi$ are primitive) so that we would be done in the case $l \geq k-l+1$ as well. This leaves only the case $k-l=l$ which we have excluded by the hypothesis $l \neq k / 2$.

Note that Theorem 4 gives no information when $l=k / 2$. However, as was done in [5] when $N=1$, one could attempt to give a direct ad hoc argument when $l=k / 2$. We sketch the strategy now assuming for simplicity that $l \geq 3$. The question is whether, in the notation of Theorem 2 , one can have a relation of the form (up to a constant)

$$
f_{k / 2}\left(Q^{\prime} z, \psi_{1}, \psi_{2}\right) f_{k / 2}\left(Q z, \chi_{1}, \chi_{2}\right)=f
$$

with $f$ an a.e. eigenform. There are two cases to consider now. Either both the Eisenstein series on the left hand side of (15) do not vanish at a common cusp (in which case $f$ is an Eisenstein series) or not (in which case $f$ is a cusp form).

In the former case, we may always twist by an Atkin-Lehner involution (at least if $N$ is square-free) to assume that the two Eisenstein series above do not vanish at $\infty$. Thus we may assume that $\psi_{1}=\chi_{1}=1$. If we normalize all the $q$-expansions so that the constant terms of each of the eigenforms in (15) is 1 , an inspection of the $q$ term yields

$$
\frac{1}{B_{k / 2, \psi}}+\frac{1}{B_{k / 2, \chi}}=\frac{2}{B_{k, \psi \chi}} \text {. }
$$

Presumably, this relation does not hold very often, in which case neither can the relation (15). For instance when $N=1$ the relation (16) becomes

$$
B_{k}=B_{k / 2}
$$

with $k \equiv 0 \bmod 4$, which only holds for $k=8\left(B_{8}=B_{4}\right)$, explaining why $E_{4}^{2}=E_{8}$ is the unique identity of the form $E_{k / 2}^{2}=E_{k}$ where $E_{k}$ is the unique normalized Eisenstein series of weight $k$.

In the latter case $\left(\psi_{1} \neq 1\right.$ or $\left.\chi_{1} \neq 1\right)$ a similar argument might be carried out by looking at, say, the $q^{2}$ term of (15).

8. Non-vanishing of $L$-functions. As an application of some of the arguments presented in the previous sections we give a criterion for the non-vanishing of twists of standard $L$-functions at the center of the critical strip. More precisely we prove:

TheOREM 5. Let $N \geq 1$ be an arbitrary integer and let $k \geq 6$ be an even integer. Assume that $\psi$ and $\chi$ are $\bmod N$ Dirichlet characters such that $\psi$ 
and $\psi \chi$ are primitive. If

$$
E_{k / 2, \bar{\psi}} \cdot E_{k / 2, \bar{\chi}} \notin \mathcal{E}_{k}(N, \psi \chi),
$$

then there is a primitive form $f \in S_{k}(N, \psi \chi)$ such that

$$
L(k / 2, f \otimes \psi) \neq 0 .
$$

Proof. This follows immediately from the proof of Proposition 4. Set $g=E_{k / 2, \bar{\psi}}=f_{k / 2}(z, 1, \psi)$. Then the hypothesis (18) and the fact that $\psi \chi$ is primitive implies that $\operatorname{dim} S_{k}^{\text {new }}(N, \chi \psi) \geq 1$. Further (18) implies that there exists a primitive form $f_{c} \in S_{k}^{\text {new }}(N, \chi \psi$ ) (for some primitive form $\left.f \in S_{k}^{\text {new }}(N, \bar{\chi} \bar{\psi})\right)$ such that $\left(f_{c}, g E_{k / 2, \bar{\chi}}\right) \neq 0$. Then (13) and (14) imply that $L(k / 2, f \otimes \psi) \neq 0$, as desired.

In special cases, (18) is easy to check. For instance, various authors have already observed that the level one version of Theorem 5 can be used to prove:

Corollary 2 ([7], [3]). If $k \equiv 0 \bmod 4$ and $k>8$ then there exists a primitive cusp form of level $N=1$ and weight $k$ such that $L(k / 2, f) \neq 0$.

Proof. As already noted, (17) fails for such $k$ implying that (18) holds.

Explicit versions of Theorem 5 may be deduced for arbitrary level. The following corollary gives an example when $N$ is prime.

Corollary 3. Say that $N=p$ is prime and that $k \geq 6$ is an even integer. Let $\psi$ and $\alpha$ be distinct primitive characters of level $p$. Suppose that the system of equations

$$
\begin{aligned}
& {\left[\begin{array}{cc}
-B_{k, \alpha} /(2 k) & 0 \\
1 & 1 \\
1+\alpha(2) 2^{k-1} & \alpha(2)+2^{k-1}
\end{array}\right]\left[\begin{array}{l}
x \\
y
\end{array}\right]} \\
& =\left[\begin{array}{c}
\frac{B_{k / 2, \psi}}{k} \cdot \frac{B_{k / 2, \bar{\psi} \alpha}}{k} \\
-\frac{B_{k / 2, \psi}}{k}-\frac{B_{k / 2, \bar{\psi} \alpha}}{k} \\
1-\frac{B_{k / 2, \psi}}{k}\left(1+\bar{\psi} \alpha(2) 2^{k / 2-1}\right)-\frac{B_{k / 2, \bar{\psi} \alpha}}{k / 2}\left(1+\psi(2) 2^{k / 2-1}\right)
\end{array}\right]
\end{aligned}
$$

is inconsistent. Then there exists a primitive cusp form $f \in S_{k}(p, \alpha)$ such that $L(k / 2, f \otimes \psi) \neq 0$.

Proof. We apply Theorem 5 with $\chi$ replaced by $\bar{\psi} \alpha$. The Eisenstein series $f_{k}(z, 1, \alpha)$ and $f_{k}(z, \alpha, 1)$ give a basis of $\mathcal{E}_{k}(N, \alpha)$. An inspection of the first three terms in the $q$-expansion of the relation 


$$
E_{k / 2, \bar{\psi}} \cdot E_{k / 2, \psi \bar{\alpha}}=x \cdot f_{k}(z, 1, \alpha)+y \cdot f_{k}(z, \alpha, 1)
$$

gives the system of equations (19) for unknowns $x$ and $y$. Thus if the system (19) is inconsistent, (18) holds, and the corollary follows.

9. Examples. In this section we give examples of a.e. eigenforms that are products of a.e. eigenforms of smaller weight. We do not impose any restriction on the weights of the eigenforms or on their level since we expect that the results proven in this paper should generalize. In particular we do not expect any such relations in Case A, even for arbitrary level.

So we start with Case B. Let us assume that $g$ is a newform. Recall that Theorem 3 does not apply when the dimension of the space $S$ of cusp forms in which the product lies has new part of dimension one. In particular it does not apply if $S$ has dimension one. Table 2 lists all the spaces $S_{k}(N, \chi)$ of dimension one, for $k>1$, along with a decomposition, if possible, of a generator $\Delta_{k}(N, \chi)$ of $S_{k}(N, \chi)$ into a product of an a.e. eigen-cusp form and an a.e. eigen-Eisenstein series of smaller weight.

Here are some remarks on reading Table 2:

- We have written $\Delta_{k}(N)$ for the generator $\Delta_{k}(N, \chi)$ when $\chi=1$. When possible we have identified it in terms of the Ramanujan Delta function $\Delta(z)$.

- All the identities hold only up to a constant (for which see Theorem 2).

- The notation used for the nebentypus $\chi$ is the same as that used in the modular forms calculator HECKE (more about HECKE below) and is self explanatory. Warning: this notation only identifies $\chi$ up to Galois conjugacy, but this suffices for our purposes since $\operatorname{dim} S_{k}(N, \chi)=\operatorname{dim} S_{k}\left(N, \chi^{\sigma}\right)$ for $\sigma \in \operatorname{Gal}(\overline{\mathbb{Q}} / \mathbb{Q})$.

- Even when the Galois conjugacy class of $\chi$ is larger than one, we have listed only the identity for $\chi$; the identities for the conjugate characters are obtained in an obvious manner.

- Since there are no general formulas for the dimensions of the weight 1 spaces (and we are not aware of any tables in the literature) we have omitted these spaces from the table. For this reason, we have also not listed the weight 2 spaces of dimension one (there are twenty-nine such spaces up to Galois conjugacy whose levels range between $N=11$ and $N=49$ ).

Table 2 was compiled, in part, manually, using well known formulas for the dimensions of spaces of cusp forms. It was completed using the modular forms calculator HECKE designed by W. Stein which computes dimensions using the trace formula in Hijikata [6]. Stein has also tabulated the dimensions of the spaces $S_{k}(N, \chi)$ for $N \leq 400$ using the alternative formula given in Cohen-Oesterlé [2]. The package HECKE as well as various tables can be found at Stein's home page [10]. 
Table 2. Tuples $(k, N, \chi)$ with $\operatorname{dim} S_{k}(N, \chi)=1$

\begin{tabular}{|c|c|c|c|}
\hline Weight & Level & Nebentypus & Product of cusp form and Eisenstein series? \\
\hline$k=1$ & $N=? ?$ & & \\
\hline$k=2$ & Twenty-nine & Cases & \\
\hline$k=3$ & $N=7$ & $\chi=\chi_{2}$ & \\
\hline$k=3$ & $N=8$ & $\chi=\chi_{2,2}$ & \\
\hline$k=3$ & $N=9$ & $\chi=\chi_{6}$ & \\
\hline$k=3$ & $N=10$ & $\chi=\chi_{4}$ & \\
\hline$k=3$ & $N=11$ & $\chi=\chi_{2}$ & $\Delta_{3}\left(11, \chi_{2}\right)=\Delta_{2}(11) E_{1, \chi_{2}}$ \\
\hline$k=3$ & $N=11$ & $\chi=\chi_{10}$ & $\Delta_{3}\left(11, \chi_{10}\right)=\Delta_{2}(11) E_{1, \chi_{10}}$ \\
\hline$k=3$ & $N=12$ & $\chi=\chi_{1,2}$ & \\
\hline$k=3$ & $N=13$ & $\chi=\chi_{12}$ & $\Delta_{3}\left(13, \chi_{12}\right)=\Delta_{2}\left(13, \chi_{6}\right) E_{1, \chi_{4}}$ \\
\hline$k=3$ & $N=16$ & $\chi=\chi_{2,1}$ & $\begin{array}{l}\Delta_{3}\left(16, \chi_{2,1}\right)=\Delta_{2}\left(16, \chi_{1,4}\right) E_{1, \bar{\chi}_{2,4}} \\
\quad=\Delta_{2}\left(16, \bar{\chi}_{1,4}\right) E_{1, \chi_{2,4}}\end{array}$ \\
\hline$k=4$ & $N=5$ & $\chi=1$ & $\Delta_{4}(5)=(\Delta(z) \Delta(5 z))^{1 / 6}$ \\
\hline$k=4$ & $N=6$ & $\chi=1$ & $\Delta_{4}(6)=(\Delta(z) \Delta(2 z) \Delta(3 z) \Delta(6 z))^{1 / 12}$ \\
\hline$k=4$ & $N=7$ & $\chi=1$ & $\Delta_{4}(7)=\Delta_{3}\left(7, \chi_{2}\right) E_{1, \chi_{2}}$ \\
\hline$k=4$ & $N=7$ & $\chi=\chi_{3}$ & $\Delta_{4}\left(7, \chi_{3}\right)=\Delta_{3}\left(7, \chi_{2}\right) E_{1, \chi_{6}}$ \\
\hline$k=4$ & $N=8$ & $\chi=1$ & $\Delta_{4}(8)=\Delta_{3}\left(8, \chi_{2,2}\right) E_{1, \chi_{2,2}}$ \\
\hline$k=4$ & $N=9$ & $\chi=1$ & $\Delta_{4}(9)=\Delta_{3}\left(9, \chi_{6}\right) E_{1, \bar{\chi}_{6}}$ \\
\hline$k=5$ & $N=4$ & $\chi=\chi_{2}$ & \\
\hline$k=5$ & $N=5$ & $\chi=\chi_{4}$ & \\
\hline$k=5$ & $N=7$ & $\chi=\chi_{2}$ & $\Delta_{5}\left(7, \chi_{2}\right)=\Delta_{3}\left(7, \chi_{2}\right) E_{2}^{(7)}$ \\
\hline$k=6$ & $N=3$ & $\chi=1$ & $\Delta_{6}(3)=(\Delta(z) \Delta(3 z))^{1 / 4}$ \\
\hline$k=6$ & $N=4$ & $\chi=1$ & $\Delta_{6}(4)=\Delta_{5}\left(4, \chi_{2}\right) E_{1, \chi_{2}}$ \\
\hline$k=6$ & $N=5$ & $\chi=1$ & $\Delta_{6}(5)=\Delta_{4}(5) E_{2}^{(5)}$ \\
\hline$k=7$ & $N=3$ & $\chi=\chi_{2}$ & $\Delta_{7}\left(3, \chi_{2}\right)=\Delta_{6}(3) E_{1, \chi_{2}}$ \\
\hline$k=8$ & $N=2$ & $\chi=1$ & $\Delta_{8}(2)=(\Delta(z) \Delta(2 z))^{1 / 3}$ \\
\hline$k=8$ & $N=3$ & $\chi=1$ & $\Delta_{8}(3)=\Delta_{7}\left(3, \chi_{2}\right) E_{1, \chi_{2}}=\Delta_{6}(3) E_{2}^{(3)}$ \\
\hline$k=10$ & $N=2$ & $\chi=1$ & $\Delta_{10}(2)=\Delta_{8}(2) E_{2}^{(2)}$ \\
\hline$k \geq 12$ & $N=1$ & $\chi=1$ & Twelve identities: see display (5) of [5] \\
\hline
\end{tabular}

Let us now turn our attention to Case C. We impose the restriction that both $g$ and $h$ have primitive nebentypus (but allow the case $l=k / 2$ ). Recall that Theorem 4 does not treat the following exceptional cases:

(i) $g h \in S_{k}(N, \psi \bar{\omega})$ and $\operatorname{dim} S_{k}^{\text {new }}(N, \psi \bar{\omega}) \leq 1$,

(ii) $g h \in \mathcal{E}_{k}(N, \psi \bar{\omega})$ and $\operatorname{dim} S_{k}^{\text {new }}(N, \psi \bar{\omega})=0$. 
Let us discuss each of these cases in turn.

In case (i) let us suppose that $\operatorname{dim} S_{k}(N, \psi \bar{\omega})=1$. With this restriction clearly $g h$ is an a.e. eigenform. Many of the entries in Table 2 can be written as a product of Eisenstein series. It would be too time consuming to give an exhaustive list of these relations. We content ourselves with an example: the cusp form $\Delta_{6}(3)$, which could not be written as a product of an a.e. eigencusp form and an a.e. eigen-Eisenstein series within the limits of Table 2, can be written as the product of two a.e. eigen-Eisenstein series. Namely, we have

$$
\Delta_{6}(3)=f_{3}\left(z, \chi_{2}, 1\right) f_{3}\left(z, 1, \chi_{2}\right) .
$$

As for case (ii), one would have to go through the (finite) list of tuples $(k, N, \chi)$ for which there are no primitive cusp forms, identifying by inspection which elements of $\mathcal{E}_{k}(N, \chi)$ can be written as products of Eisenstein series of lower weight. Again it would be too time consuming to tabulate all such relations completely. We merely point out that Table 1 gives an example of such a relation when the level is larger than one. Indeed, if $p \equiv 3 \bmod 4$ and $S_{2}\left(\Gamma_{0}(p)\right)=0$, that is, when $p=3$ or 7 , we have

$$
E_{1, \chi_{-p}}^{2}=E_{2}^{(p)}
$$

up to a constant. Here $\chi_{-p}$ is the odd quadratic character of conductor $p$. In the case $p=3$, this was already reflected in the entry $(8,3,1)$ of Table 2 (there $\chi_{-3}$ was called $\chi_{2}$ ).

\section{References}

[1] A. O. L. Atkin and W. C. W. Li, Twists of newforms and pseudo-eigenvalues of $W$-operators, Invent. Math. 48 (1978), 221-243.

[2] H. Cohen et J. Oesterlé, Dimensions des espaces de formes modulaires, in: Modular Functions of One Variable, VI (Bonn, 1976), Lecture Notes in Math. 627, Springer, Berlin, 1977, 69-78.

[3] B. Conrey and D. Farmer, Hecke operators and the non-vanishing of L-functions, in: Topics in Number Theory (University Park, PA, 1997), Math. Appl. 467, Kluwer, Dordrecht, 1999, 143-150.

[4] W. Duke, When is the product of two Hecke eigenforms an eigenform?, in: Number Theory in Progress, Vol. 2 (Zakopane, 1997), de Gruyter, Berlin, 1999, 737-741.

[5] E. Ghate, On monomial relations between Eisenstein series, J. Ramanujan Math. Soc. 15 (2000), 71-79.

[6] H. Hijikata, Explicit formula of the traces of Hecke operators for $\Gamma_{0}(N)$, J. Math. Soc. Japan 26 (1974), 56-82.

[7] W. Kohnen and D. Zagier, Values of L-series of modular forms at the center of the critical strip, Invent. Math. 64 (1981), 175-198.

[8] T. Miyake, Modular Forms, Springer, 1989. 
[9] G. Shimura, The special values of the zeta functions associated with cusp forms, Comm. Pure. Appl. Math. 29 (1976), 783-804.

[10] W. Stein, HECKE, http://modular.fas.harvard.edu.

School of Mathematics

Tata Institute of Fundamental Research

Homi Bhabha Road

Mumbai, 400005, India

E-mail: eghate@math.tifr.res.in

Received on 22.1.2001

and in revised form on 16.5.2001 